This item was submitted to Loughborough's Research Repository by the author.

Items in Figshare are protected by copyright, with all rights reserved, unless otherwise indicated.

\title{
Stochastic security-constrained generation expansion planning methodology based on a generalized line outage distribution factors
}

PLEASE CITE THE PUBLISHED VERSION

https://doi.org/10.1109/PTC.2017.7981191

PUBLISHER

(C) IEEE

VERSION

AM (Accepted Manuscript)

\section{PUBLISHER STATEMENT}

This work is made available according to the conditions of the Creative Commons Attribution-NonCommercialNoDerivatives 4.0 International (CC BY-NC-ND 4.0) licence. Full details of this licence are available at: https://creativecommons.org/licenses/by-nc-nd/4.0/

\section{LICENCE}

CC BY-NC-ND 4.0

\section{REPOSITORY RECORD}

Hinojosa, Victor H., and Francisco M. Gonzalez-Longatt. 2019. "Stochastic Security-constrained Generation Expansion Planning Methodology Based on a Generalized Line Outage Distribution Factors”. figshare. https://hdl.handle.net/2134/25768. 


\section{Stochastic security-constrained generation expansion planning methodology based on a generalized line outage distribution factors}

\author{
Victor Hinojosa \\ Department of Electrical Engineering \\ Universidad Técnica Federico Santa María \\ Av. España 1680, Valparaíso - Chile \\ Email: victor.hinojosa@usm.cl \\ Telephone: (56 32) 265-4357
}

\author{
Francisco Gonzalez-Longatt \\ School of Electric, Electronic and Systems Engineering \\ Loughborough University \\ Loughborough - United Kingdom \\ Email: fglongatt@fglongatt.org
}

\begin{abstract}
In this study, the authors proposes to develop an efficient formulation in order to figure out the stochastic securityconstrained generation capacity expansion planning (SC-GCEP) problem. The main idea is related to directly compute the line outage distribution factors (LODF) which could be applied to model the $N-m$ post-contingency analysis. In addition, the post-contingency power flows are modeled based on the LODF and the partial transmission distribution factors (PTDF). The PTDF-based generation capacity planning formulation has been reformulated in order to include the post-contingency constraint solving both pre- and post-contingency constraints simultaneously. The methodology includes in the optimization problem the load uncertainty using a two-stage multi-period model, and a $K$-means clustering technique is applied to reduce the load scenarios. The main advantage of this methodology is the feasibility to quickly compute the post-contingency factors especially with multiple-line outages $(N-m)$. This idea could speed up contingency analyses and improve significantly the securityconstrained analyses applied to stochastic GCEP problems. It is conducted several experiments with two electrical power systems in order to validate the performance of the proposed formulation.

Index Terms-Linear distribution factors; securityconstrained; stochastic programming; two-stage problem; uncertainty;
\end{abstract}

\section{INTRODUCTION}

The GCEP is an issue of determining, i) how many capacity to add, ii) what type of generation is needed, and iii) when and where to locate new generating units so that the load customers are adequately supplied for a foreseen future, typically 10-20 years.

Capacity expansion planning was first formulated as an optimization problem in 1957 [1], but it was only after development of computing and decomposition techniques that capacity expansion plans for simple models of real power systems could be obtained [2]. Assuming that the electricity market is centrally operated (e.g. Chile situation), and that the generation companies do not have the ability to accomplish local market power, the GCEP optimization problem can be formulated as a cost minimization in which operational costs are modeled using a DC-network through an optimal power flow (DC-OPF) model [3], [4]. Additionally, the objective of security analysis is to determine a minimum operation point where the system is kept in a normal state after a major disturbance such as transmission line, transformer or generator outage [5]. The preventive security-constrained formulation minimizes some cost function by considering only the normal case control variables that are feasible for both normal and contingency conditions. The reader is referred to [6], [7] for a recent literature review.

In the literature review, the most accepted model used by researchers is the so-called DC-based model. In electrical power systems, this classical formulation has been broadly used by researchers to solve different operation and planning problems [8]-[11]. In addition, deterministic models; i.e. assuming perfect foresight, have been widely studied, and several methods to solve the GCEP problem have been proposed using dynamic programming [12], [13] and branchand-bound methods [3], [6], [14], [15]. Another very important factor in operation and planning problems is the modeling of uncertainty in input variables. Actually, stochastic generation planning models have been investigated by different authors [10], [15]-[18]. In these references, the uncertainty of input variables has been included with different approaches.

In this study, it is modeled the load uncertainty using the two-stage stochastic mixed-integer problem (SMIP) [19] based on non - anticipativity constraints. The model uses the $K-$ means clustering algorithm [20] in order to reduce the load scenarios [10] keeping the associated optimization problem smaller; furthermore, the clustering algorithm computes their respective probability for its use in the SMIP objective function. This approach has been used by several researchers [10], [15].

This study is based on the previous methodology carried out by Hinojosa [4]. The methodology uses linear distribution factors in order to model the pre- and post-contingency constraints simultaneously. It should be pointed out that prominent results were obtained modeling the pre- and the postcontingency $(N-1$ and $N-2)$ analyses. On the other hand, 
the framework to model the post-contingency constraints has some complications when the algorithm needs to model and include more that two contingencies. Since each contingency requires separate LODFs, a quick calculation of LODFs, especially with multiple-line outages, could speed up contingency analyses and improve significantly the security analyses of power systems [21].

The proposed methodology has not been implemented in the technical literature. Moreover, there is no evidence about the performance in the simulation time; therefore, it would be very interesting to conduct several analyses with large-scale power systems and increasing the number of load scenarios in order to validate the simulation time.

\section{DC-BASED PREVENTIVE SECURITY-CONSTRAINED PROBLEM}

The uses of scenarios to represent the uncertainty allows to formulate the SMIP starting from a deterministic MILP formulation as the minimization of the expected value of the deterministic objective function, which turns out to be the weighted sum of the objective function for $S$ possible realization of the uncertainty parameter (scenarios) with their respective probabilities of occurrence $\left(\omega_{s}\right)$ [22]. In the proposed formulation, the first stage variables (investment decisions of generation capacity) are the decisions to make under uncertainty and are the same for all different realizations of the uncertain parameter (long-term load forecasting). The second stage variables (power generation of each unit) must supply the load customers for each scenario considering a DCnetwork through a security-constrained DC-OPF problem.

From a central planner point of view, stochastic GCEP problem can be formulated as the minimization of the following terms: 1) investment cost from candidate power units; 2) fixed operational \& maintenance cost from candidate power units; 3) variable operational cost from existing and candidate power units; and 4) unserved energy cost. Moreover, it is assumed that $m$ potential contingencies $(N-m)$ could be added in the operational problem in order to model a securityconstrained problem. Note that it is supposed that the active power generation is the same for both the pre-contingency $(N-0)$ and the post-contingency analysis (for instance $N-1$ or $N-2$ ). Notice that only one equation is needed to maintain the pre- and post-contingency balance. More details about pre- and post-contingency transmission modeling using linear factors could be reviewed in [3], [4], [23], [24].

\section{A. Mathematical formulation}

The DC-based transmission power losses have not been included in the optimization problem; therefore, the optimization problem can be formulated as follows:

$$
\begin{gathered}
\sum_{s \in S} \omega_{s}\left\{\sum_{t \in T} \alpha_{t}[\underbrace{\sum_{g \in B}\left(I_{g} \cdot\left(n_{s, t, g}-n_{s, t-1, g}\right)+O M_{g} \cdot n_{s, t, g}\right) \cdot P_{g}^{M}}_{\text {fixed (investment }+O \& M) \text { cost }}+\right. \\
\underbrace{\sum_{y \in Y} \sum_{g \in(B \cup E)} F_{g} \cdot h_{y} \cdot P_{s, t, y, g}}_{\text {variable (operational) cost }}+\underbrace{\left.V o L L \cdot h_{y} \cdot \sum_{g \in R} P_{s, t, y, g}\right]}_{\text {unserved energy cost }}\}
\end{gathered}
$$

s.t.

Capacity reserve margin: planning reserve margin is designed to measure the amount of generation capacity available to meet expected demand in planning horizon. Coupled with probabilistic analysis, calculated planning reserve margins have been an industry standard used by planners for decades as a relative indication of adequacy (NERC).

$$
\sum_{g \in B} P_{g}^{M} \cdot n_{s, t, g}+\sum_{g \in(E \cup r)} P_{g}^{M} \geq d_{s, t}^{\text {peak }}+S R
$$

Load balance: in spite of the fact that there are three balance conditions $(N-0, N-1$ and $N-2)$, only one equation is needed since active power generation for each condition is the same. This equation will be called global power balance equation (3), and it is used to satisfy the load for the pre- and post-contingency cases simultaneously.

$$
\sum_{g \in(B \cup E \cup r)} h_{y} \cdot P_{s, t, y, g}=D_{s, t, y}
$$

Transmission limits: the power flow through transmission elements (either lines or transformers) should not violate its respective capability regarding normal $(L), N-1(L-1)$ and $N-m(L-m)$ conditions.

$$
\begin{gathered}
\left|G^{N-0} \cdot\left(P_{s, t, y, k}-d_{s, t, y, k}\right)\right| \leq f_{l}^{M} \quad \forall l \in L \\
\left|G^{N-m} \cdot\left(P_{t, y, k}-d_{t, y, k}\right)\right| \leq f_{l}^{M} \quad \forall l \in L-m
\end{gathered}
$$

Nonlinear transformation: because of the nonlinear transformation, it is necessary to include a inequality constraint to limit the active power supplied by candidate units $g$.

$$
P_{s, t, y, g} \leq P_{g}^{M} \cdot n_{s, t, g}
$$

Sequential installation: these constraints represent the logical relationship for the construction status of different generating units within the planning horizon.

$$
n_{s, t-1, g} \leq n_{s, t, g}
$$

Maximum power units: the maximum (accumulated) number of power units is limited considering a maximum number of generators to be built. 


$$
n_{s, t, g}-n_{s, t-1, g} \leq n_{g}^{M}
$$

Non-anticipativity constraints: the SMIP is solved using non - anticipativity constraints, which force the first stage variables (investment decisions of generation capacity) to be the same for each scenario.

$$
\begin{gathered}
n_{1, t, g}=n_{s, t, g} \\
\forall s \in S, \forall t \in T, \forall y \in Y
\end{gathered}
$$

where $S, G, L, T$, and $Y$ are load scenarios, set of all generators, transmission elements, years in the planning horizon, and load duration curve (LDC) blocks, respectively. $B \subset G, E \subset G$ and $r \subset G$ are the indexes of generators with building decisions, actual generators and virtual generators (load shedding). $I_{g}$ is the investment cost of generator $g, O M_{g}$ is the operation and maintenance cost, $V o L L$ is the value of lost load, $\alpha_{t}$ is the discount economic factor, $h$ is the number of hours in LDC block; $S R$ is spinning reserve; $d^{\text {peak }}$ is peak load; $D$ is the total load; and $f_{l}^{M}$ is the maximum power flow through line $l . P_{s, t, y, g}$ is the total active power supplied by the plant $g$ (block $y$, planning stage $t$, scenario $s$ ), $P_{g}^{M}$ is the maximum active power, $n_{s, t, g}$ is the number of units to build, and $n_{g}^{M}$ is the maximum number of units with building decisions.

\section{B. Improved method to compute directly the LODFs}

In [3], [4], the pre- and post-contingency transmission constraints are formulated using the partial transmission distribution factors (PTDF) and LODF.

1) Pre-contingency transmission limits: For the precontingency transmission constraint, the following matrix is defined $G^{N-0}=P T D F^{N-0}$.

2) Post-contingency transmission limits: In this study, it is used the LODF definition [5] in order to compute the postcontingency transmission constraints (9):

$$
f_{l}^{N-1}=f_{l}^{N-0}+L O D F_{l, o 1}^{N-0} \cdot f_{o 1}^{N-0} \forall l \in L-1
$$

where $f_{l}^{N-0}$ and $f_{o 1}^{N-0}$ are the pre-contingency power flows on $l-t h$ line and line $o 1$, respectively, and $f_{l}^{N-1}$ is the $N-1$ power flow (post-contingency) on $l-t h$ line with line $o 1$ out.

Using the PTDF-based formulation and equation (9), it is possible to compute the $N-1(m=1)$ linear factor $(10)$ :

$$
G^{N-1}=P T D F_{l}^{N-0}+L O D F_{l, o 1}^{N-0} * P T D F_{o 1}^{N-0}
$$

On the other hand, the $N-2(m=2)$ analysis is solved using the following expressions (11):

$$
f_{l}^{N-2}=f_{l}^{N-1}+L O D F_{l, o 2}^{N-1} \cdot f_{o 2}^{N-1} \forall l \in L-2
$$

where $f_{o 2}^{N-1}=f_{o 2}^{N-0}+L O D F_{o 2, o 1}^{N-0} \cdot f_{o 1}^{N-0}$.
Using the PTDF-based formulation and equation (11), it is possible to compute the $N-2(m=2)$ linear factor (12):

$$
\begin{gathered}
G^{N-2}=P T D F_{l}^{N-0}+\left[\left(L O D F_{l, o 1}^{N-0}+L O D F_{l, o 2}^{N-l} *\right.\right. \\
\left.\left.L O D F_{o 2, o 1}^{N-0}\right) * P T D F_{o 1}^{N-0}\right]+L O D F_{l, o 2}^{N-l} * P T D F_{o 2}^{N-0}
\end{gathered}
$$

where line $o 1$ and $o 2$ are out simultaneously.

These expressions $\left(G^{N-0}, G^{N-1}\right.$ and $\left.G^{N-2}\right)$ are used for computing the pre- and post-contingency power flows in the transmission network.

\section{Post-contingency transmission limits using a generalized formulation}

In spite of the fact that the $N-3(m=3)$ could be mathematically formulated, it is proposed to apply the algorithm and methodology proposed in [21] in order to compute the $N-m$ power flows using the LODFs 13):

$$
\begin{gathered}
f_{l}^{N-m}=f_{l}^{N-0}+L O D F_{l, O} \cdot f_{O}^{N-0} \forall l \in L-m \\
; L O D F_{l, O}=P T D F_{l}^{N-0} * A_{O_{r}}^{T} *\left(I_{O}-P T D F_{O}^{N-0} * A_{O_{r}}^{T}\right)^{-1}
\end{gathered}
$$

where $O$ represents the set of lines on outage, $A_{O_{r}}^{T}$ is the reduced bus-to-tripped line incidence matrix, and $I_{O}$ is the identity matrix for the outage lines. Note that it is changed the original notation in order to show clearly the classical PTDF definition; i.e., $L O D F_{l, O}=P T D F_{l} \cdot A_{O_{r}}^{T}$ and $L O D F_{O, O}=$ $P T D F_{O} \cdot A_{O_{r}}^{T}$.

Using the PTDF-based formulation $\left(G^{N-0}\right)$ and previous $L O D F_{l, O}$ definition, it is possible to change the precontingency power flows considering only the PTDF matrix and the net power injections. As a result, the $N-m$ transmission constraints can be computed using (14).

$$
\left|G^{N-m} \cdot\left(P_{t, y, k}-d_{t, y, k}\right)\right| \leq f_{l}^{M} \forall l \in L-m
$$

where $G^{N-1}=P T D F_{l}^{N-0}+L O D F_{l, O} * P T D F_{O}^{N-0}$.

Arranging the mathematical terms, the final expression used to compute the power flow through $l-t h$ line when $O$-th are out is as follows (15):

$$
\begin{gathered}
G^{N-m}=P T D F_{l}^{N-0} *\left[I_{b-1}+A_{O_{r}}^{T} *\left(I_{O}-P T D F_{O}^{N-0} *\right.\right. \\
\left.\left.A_{O_{r}}^{T}\right)^{-1} * P T D F_{O}^{N-0}\right]
\end{gathered}
$$

where $I$ is the identity matrix, and $(b)$ is the number of buses.

\section{Simulation RESUltS}

Two power systems are considered to inspect and verify the proposed formulation. It is conducted several experiments in order to validate the formulation, and determine the performance in the simulation time. In addition, the optimization problem was solved using $M A T L A B$ and Gurobi on a computer with the following characteristics: Intel Core i7 3930 (3.20GHz) with RAM 32GB. 


\section{A. 6-bus test power system}

The first test system used to validate and illustrate the proposed formulation is the classic 6-bus power system introduced by Wood and Wollemberg. Technical transmission data is obtained from [5], but transmission limit constraints are changed to $300[M W]$. This problem considers a $20 \%$ of capacity reserve margin; i.e. 1.2 times the yearly peak load. Also, the maximum number of power units to be built is 3 , and the $V o L L$ value is $10000[\$ / M W h]$. The interest rate used is $10 \%$. For the MILP problem, the gap used is $0.0001 \%$. Besides, tenth investment periods have been modeled in the optimization problem. Generation data for the new, virtual and existing power generation is shown in Table I. Information about the number of hours $\left(h_{t}\right)$ for each time period could be reviewed in [4].

Table I

GENERATOR DATA FOR IEEE 6-BUS TEST SYSTEM

\begin{tabular}{ccccc}
\hline Bus & $\begin{array}{c}\text { Capacity } \\
\text { (MW) }\end{array}$ & $\begin{array}{c}\text { Inv. cost } \\
(\$ / M W)\end{array}$ & $\begin{array}{c}\text { OM cost } \\
(\$ / M W)\end{array}$ & $\begin{array}{c}\text { Oper. cost } \\
(\$ / M W h)\end{array}$ \\
\hline 1 & 250 & existing unit & - & 18.50 \\
4 & 100 & existing unit & - & 14.36 \\
4 & 50 & existing unit & - & 22.11 \\
1 & 150 & 300000 & 12000 & 20.41 \\
3 & 100 & 250000 & 30000 & 25.95 \\
5 & 250 & 350000 & 36000 & 14.08 \\
$4,5,6$ & 999 & virtual unit & - & VoLL \\
\hline
\end{tabular}

1) Representation of load uncertainty: The optimization problem requires the definition of future scenarios in order to model load uncertainty. In expansion capacity planning, one of the most popular model to represent adequately the load evolution is the Geometric Brownian Motion [25]. For each case analyzed, the methodology simulates 100 scenarios (total expected behaviour) using the Geometric Brownian Motion.

Before sending the problem to the solver with all scenarios, one important step is the scenario reduction. The basic idea of the scenario reduction is to cluster similar load scenarios into groups. Accordingly, scenario reduction techniques can be employed to reduce the number of scenarios while maintaining a good approximation of the system uncertain behaviour.

The $K-$ means clustering algorithm is applied to reduce the load scenarios as well as to obtain their respective probabilities of occurrence in order to formulate the SMIP optimization problem.

For instance in Fig. 1, it is shown both the long-term load scenarios (10 years with four periods: valley, rest, mean, peak) and scenario reduction using the clustering algorithm in order to model 10 scenarios. A higher number of generated scenarios results in a better modeling of uncertainties but also the simulation time will be higher.
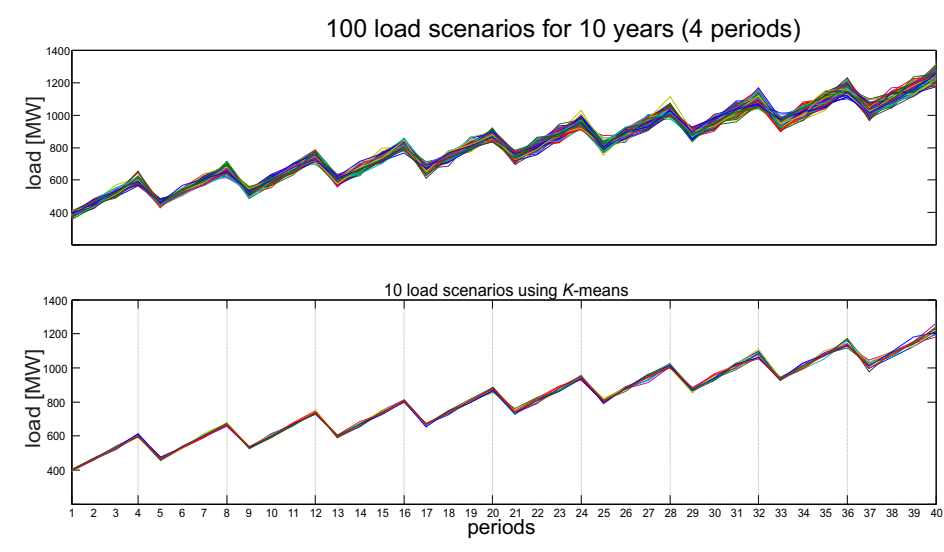

Figure 1. Uncertainty load modeling using a Geometric Brownian Motion

In this figure, it is shown the yearly load modeling. For this first test system and for each load scenario, the total load (valley, rest, mean and peak) is divided proportionally for each load bus (bus 4 , bus 5 and bus 6 ) in order to carry out the GCEP problem.

2) SMIP formulation considering a $N-2$ analysis and 5 load scenarios: A $N-2$ analysis, with line $1-2$ and line $3-6$ out, is solved. The optimal cost for the proposed formulations is $\$ 947848572.00$. The optimization problem includes one unit for bus 1 in $t_{1}$; one unit for bus 3 in $t_{8}$ and $t_{9}$; and one unit for bus 5 in $t_{1}$ and $t_{4}$. Moreover, there is not load shedding in the solution regarding all scenarios but there are forty-nine periods with congestion on transmission line $5-6$ for the $N-2$ condition.

For this case, it is decided to compare the solution obtained using the PTDF\&LODF-based formulation [4]. Both solutions are the same; therefore, it is confirmed the accuracy and optimality of the new mathematical formulation validating the LODF algorithm.

3) SMIP formulation considering a $N-m$ analysis and 5 load scenarios: A $N-3$ analysis, with line $1-2$, line $2-4$ and line $1-2$ out, is solved. The optimal cost for the proposed formulations is $\$ 963409712.71$. The optimization problem includes one unit for bus 1 in $t_{1}$ and $t_{4}$; one unit for bus 3 in $t_{6}, t_{8}$ and $t_{9}$; and one unit for bus 5 in $t_{1}$. Moreover, there is not load shedding but there are sixty-nine periods with congestion.

A $N-4$ analysis, with line $1-2$, line $2-4$, line $2-5$ and line $1-2$ out, is solved. The optimal cost for the proposed formulations is $\$ 1091668691.74$. The optimization problem includes one unit for bus 1 in $t_{3}$ and $t_{8}$; one unit for bus 3 in $t_{1}, t_{5}$ and $t_{7}$; and one unit for bus 5 in $t_{1}$. There is not load shedding but there are one hundred and thirty periods with congestion.

A $N-5$ analysis, with line $1-2$, line $1-5$, line $2-4$, line $2-5$ and line $1-2$ out, is solved. The optimal cost for the proposed formulations is $\$ 1107315292.10$. The optimization problem includes one unit for bus 1 in $t_{10}$; one unit for bus 3 in $t_{1}, t_{5}$ and $t_{7}$; and one unit for bus 5 in $t_{1}$ and $t_{3}$. There is 
not load shedding but there are one hundred and thirty periods with congestion.

These results lead to conclusion that inclusion of security criteria in the optimization problem strengthens the investment plan so that the system is kept in a normal operation state in order to supply the load customers and avoid load shedding after a major disturbance ( $N-1, N-2$, and so on).

Finally, a $N-6$ analysis, with line $1-2$, line $1-5$, line $2-4$, line $2-5$, line $2-6$ and line $1-2$ out, is solved. However, the solution has load shedding. It is worth to mentioning that there is not possible to supply the load of the customers when six lines are out simultaneously; therefore, the virtual generation (lost of load) is activated.

4) SMIP formulation considering different load scenarios: Considering the lower simulation time and using the proposed formulation, we have conducted several analyses increasing the number of load scenarios to 10 scenarios and taking into account that a higher number of scenarios results in a better modeling of load uncertainty. Table II shows simulation results for each case using the proposed formulation (PF). It is also included the objective function (FO).

Table II

SIMULATION RESULTS APPLIED TO 6-BUS POWER SYSTEM

\begin{tabular}{cccccc}
\hline model & $S$ & bus 1 & bus 3 & bus 5 & FO, $\$$ \\
\hline$P F^{N-2}$ & 5 & $t_{1}$ & $t_{8}, t_{9}$ & $t_{1}, t_{4}$ & 947848572.00 \\
$P F^{N-3}$ & 5 & $t_{1}, t_{4}$ & $t_{6}, t_{8}, t_{8}$ & $t_{1}$ & 963409712.71 \\
$P F^{N-4}$ & 5 & $t_{3}, t_{8}$ & $t_{1}, t_{5}, t_{7}$ & $t_{1}$ & 1091668691.74 \\
$P F^{N-5}$ & 5 & $t_{10}$ & $t_{1}, t_{5}, t_{7}$ & $t_{1}, t_{3}$ & 1107315292.10 \\
$P F^{N-2}$ & 10 & $t_{1}, t_{4}, t_{8}$ & $t_{6}, t_{10}$ & $t_{1}$ & 954777275.18 \\
\hline
\end{tabular}

Notice that generation plans with 5 load scenarios are different. In fact, this is the reason for the bigger objective function when a stronger post-contingency analysis is modeled in the security-constrained optimization problem.

Additionally, it can be seen that solutions are different depending on the number of load scenarios. Considering 10 scenarios, there are more power units included in the generation planning solution in comparison with 5 scenarios.

These results lead to conclusion that inclusion of security criteria in the optimization problem strengthens the investment plan; therefore, the system is kept in a safe operational state after a major disturbance $(N-m)$.

The performance of $N-m$ post-contingency analysis, scenario reduction and SMIP applied to stochastic securityconstrained GCEP could be measured evaluating a securityconstrained DC-OPF (operational problem) for all scenarios. More details could be reviewed in [4].

\section{B. 2383-bus test power system}

The Polish power system added in MATPOWER is used for testing the proposed approach applied to large-scale power systems. This study case includes 400, 220 and $110[k V]$ transmission lines, and consists of three hundred twentyseven generators and two thousand eight hundred ninetysix transmission lines. Tenth investment periods have been modeled in the optimization problem, and the maximum load in the last year is $D=26220[M W]$. This problem considers a $20 \%$ of reserve. Data for actual power units can be reviewed using MATPOWER, and data for the future generation system is provided in Table III. In addition, transmission line $6-31$ is limited to $600[M W]$. For the MILP problem, the gap used is $0.01 \%$.

Table III

GENERATOR DATA FOR FUTURE POWER UNITS

\begin{tabular}{cccccc}
\hline Bus & $P^{M}$ & $n^{M}$ & $F$ & $I$ & $O M$ \\
& $M W$ & & $U S \$ / M W h$ & $M M U S \$ / M W$ & $U S \$ / M W$ \\
\hline 1 & 100 & 2 & 170 & 2.50 & 12000 \\
3 & 130 & 3 & 140 & 0.40 & 30000 \\
95 & 50 & 3 & 40 & 1.50 & 36000 \\
123 & 250 & 3 & 165 & 3.50 & 36000 \\
754 & 150 & 2 & 160 & 2.00 & 25000 \\
795 & 150 & 3 & 90 & 1.50 & 30000 \\
799 & 250 & 2 & 145 & 3.50 & 20000 \\
800 & 100 & 3 & 190 & 2.50 & 20000 \\
833 & 75 & 4 & 185 & 0.45 & 20000 \\
861 & 90 & 3 & 90 & 1.10 & 40000 \\
899 & 80 & 3 & 40 & 1.00 & 5000 \\
990 & 150 & 3 & 150 & 3.00 & 36000 \\
997 & 120 & 2 & 140 & 2.00 & 25000 \\
998 & 100 & 3 & 175 & 0.50 & 30000 \\
999 & 180 & 3 & 120 & 1.50 & 20000 \\
\hline
\end{tabular}

For this power system test, it is decided to compare the $N-1$ and $N-2$ post-contingency analyses with the solution obtained using the PTDF\&LODF-based formulation [4]. Obtained solutions are the same. Consequently, it is confirmed the accuracy and optimality of the mathematical formulation using the proposed LODF methodology.

A $N-3$ analysis, with line $8-18$, line $18-101$ and line $238-361$ out, is solved. The optimal cost for the proposed formulations is $\$ 68715041639.31$. The optimization problem includes four units for bus 3 in $t_{8}$; three units for bus 95 in $t_{5}$; three units for bus 833 in $t_{10}$; five units for bus 861 in $t_{8}$; three units for bus 899 in $t_{2}$; and one unit for bus 998 in $t_{8}$. Besides, there is not load shedding but there are two hundred periods with congestion.

The optimization algorithm includes more power units in the generation planning. The main goal is to supply the load of the customers when a stronger contingency is affecting the security of the power system.

These simulation results are very important in order to realize the performance of the simulation when the proposed formulation is applied to large-scale power systems.

\section{CONCLUSIONS}

This study demonstrated the applicability of an improved methodolody to directly compute the LODF in order to model the $N-m$ post-contingency constraint proposed formulation solving the stochastic security-constrained GCEP problem. In addition, the post-contingency power flows are modeled based on a generalized LODF and the PTDF. The PTDF-based generation capacity planning formulation has been reformulated in order to include the post-contingency constraint solving both pre- and post-contingency constraints simultaneously. The main advantage of this methodology is the feasibility 
to quickly compute the post-contingency factors especially with multiple-line outages $(N-m)$. This idea could speed up contingency analyses and improve significantly the securityconstrained analyses applied to stochastic GCEP problems. It is conducted several experiments with two electrical power systems in order to validate the performance of the proposed formulation. It is worth to mentioning that this approach is carried out without sacrificing optimality.

Currently, authors are studying decomposition techniques (Benders) in order to apply this methodology to the stochastic security-constrained generation and transmission capacity expansion planning problem.

\section{ACKNOWLEDGEMENTS}

This study was supported in part by the Chilean National Commission for Scientific and Technological Research (CONICYT) under grant Basal FB0008, and by the Universidad Técnica Federico Santa María, Chile, under project USM 116.22.2.

\section{REFERENCES}

[1] P. Masse and R. Gibrat, "Application of linear programming to investments in the electric power industry," Manage Sci., vol. 3, no. 2, pp. 149-166, 1957.

[2] M. Pereira, L. Pinto, S. Cunha, and G. Oliveira, "A decomposition approach to automated generation/transmission expansion planning," IEEE Trans. on Power Syst., vol. PAS-104, no. 11, pp. 3074-3083, 1985.

[3] V. H. Hinojosa and J. Velásquez, "Improving the mathematical formulation of security-constrained generation capacity expansion planning using power transmission distribution factors and line outage distribution factors," Electr. Power Syst. Res., vol. 140, pp. 391-400, 2016.

[4] _ , "Stochastic security-constrained generation expansion planning based on linear distribution factors," Electr. Power Syst. Res., vol. 140, pp. 139-146, 2016.

[5] A. J. Wood and B. F. Wollenberg, Power Generation, Operation and Control. John Wiley \& Sons, 1996.

[6] G. A. Bakirtzis, P. N. Biskas, and V. Chatziathanasiou, "Generation expansion planning by milp considering mid-term scheduling decisions," Elect. Power Syst. Res., vol. 86, pp. 98-112, 2012.

[7] H. Hemmati, R. Hooshmand, and A. Khodabakhshian, "Comprehensive review of generation and transmission expansion planning," IET Proc. Gener. Transm. Distrib., vol. 7, no. 9, pp. 955-964, 2013.

[8] Y. Tohidi, F. Aminifar, and M. Fotuhi-Firuzabad, "Generation expansion and retirement planning based on the stochastic programming," Electr. Power Syst. Res., vol. 104, pp. 138-145, 2013.
[9] S. Dehghan, N. Amjady, and A. Kazemi, "Two-stage robust generation expansion planning: a mixed integer linear programming model," IEEE Trans. on Power Syst., vol. 29, no. 2, pp. 584-597, 2014.

[10] E. Gil, I. Aravena, and R. Cárdenas, "Generation capacity expansion planning under hydro uncertainty using stochastic mixed integer programming and scenario reduction," IEEE Trans. on Power Syst., vol. 30, no. 4, pp. 1838-1847, 2015.

[11] S. You, S. W. Hadley, M. Shankar, and Y. Liu, "Security-constrained expansion planning of fast-response units for wind integration," Elect. Power Syst. Res., vol. 133, pp. 209-218, 2016.

[12] R. R. Booth, "Optimal generation planning considering uncertainty," IEEE Trans. Power App. Syst., vol. PAS-91, no. 1, pp. 70-77, 1990.

[13] L. A. Poch and R. T. Jenkins, "Dynamic programming models," Energy, vol. 15 , no. 7/8, pp. 573-581, 1990.

[14] H. M. Khodr, J. F. Gomez, L. Barnique, J. H. Vivas, P. Paiva, J. M. Yusta, and A. J. Urdaneta, "A linear programming methodology for the optimization of electrical power generation schemes," IEEE Trans. on Power Syst., vol. 17, no. 3, pp. 797-802, 2002.

[15] I. Aravena, R. Cárdenas, E. Gil, V. Hinojosa, J. C. Araneda, and P. Reyes, "Co-optimization of generation and transmission investment decisions under hydro uncertainty using stochastic mixed-integer programming," in Proc. 10th Latin-American Congr. Elect. Power Gen. Transm. Distrib. (CLAGTEE), no. Viña del Mar, Chile, pp. 1-8, 2013.

[16] B. Mo, J. Hegge, and I. Wangensteen, "Stochastic generation expansion planning by means of stochastic dynamic programming," IEEE Trans. on Power Syst., vol. 6, no. 2, pp. 662-668, May 1991.

[17] A. P. Sanghvi and I. H. Shavel, "Strategic planning for power system reliability and vulnerability: An optimization model for resource planning under uncertainty,' IEEE Trans. Power App. Syst., vol. PAS-101, no. 6, pp. 1420-1429, Jun 1982.

[18] R. R. Booth, "Optimal generation planning considering uncertainty," IEEE Trans. on Power Syst., vol. 27, no. 1, pp. 433-441, Feb 2012.

[19] R. O’Neill, E. Krall, K. Hedman, and S. Oren, "A model and approach to the challenge posed by optimal power systems planning," Math. Program., Series B, vol. 140, no. 2, pp. 239-266, 2013.

[20] J. C. Bezdek, "Fuzzy mathematics in pattern classification," Ph.D. dissertation, Center Appl. Math., Cornell Univ., Ithaca, N.Y., 1973.

[21] G. Jiachun, F. Yong, L. Zuyi, and M. Shahidehpour, "Direct calculation of line outage distribution factors," IEEE Trans. Power App. Syst., vol. 24, no. 3, pp. 1633-1634, 2009.

[22] J. Birge and F. Louveuax, Introduction to Stochastic Programming. ser. Springer Series in Operations Reserach and Financial Engineering, Springer-Verlag, 2011.

[23] F. Gonzalez-Longatt and J. Rueda, Power Factory Applications for Power System Analysis. Springer, 2014.

[24] V. H. Hinojosa, O. Ticuna, and G. Gutierrez, "Improving the mathematical formulation of the unit commitment with transmission system constraints," IEEE Latin Am. Trans., vol. 14, no. 2, pp. 773-781, 2016.

[25] Y. Y. Liang, "Demand modeling with geometric brownian motion process," Technical report NTU-IEChou-2003-T001, May, 2003. 